\title{
Electrochemical Determination of Alkaline Phosphatase as a Potential Marker of Reperfusion Injury
}

\author{
Shuang Qin ${ }^{1 *}$, Keshi Wang ${ }^{2}$, Xinxin Ma ${ }^{3}$,Wangcheng Xiong ${ }^{1}$, Zhongyi Yue ${ }^{1}$ and Minliang Chen ${ }^{4}$ \\ ${ }^{1}$ Department of General Surgery, The First Affiliated Hospital of Xinxiang Medical College, \\ Xinxiang, 453000, China. \\ ${ }^{2}$ The First Clinical College, Xinxiang Medical College, Xinxiang, 453000, China \\ ${ }^{3}$ The Third Clinical College, Xinxiang Medical College, Xinxiang, 453000, China \\ ${ }^{4}$ First Affiliated Hospital, Chinese Pla Medical College, Beijing, 100048, P.R. China. \\ *E-mail: chenminliang98@ foxmail.com
}

doi: $10.20964 / 2017.10 .59$

Received: 29 May 2017 / Accepted: 7 August 2017 / Published: 12 September 2017

Currently, there are no well-defined markers for distinguishing ischaemia from reperfusion injury. In this study, the practicality of a diagnostic health sensor was examined via the investigation of voltammetric alkaline phosphatase (ALP) detection. An indium tin oxide (ITO) film on glass was employed in the fabrication of an electrochemical sensor via facile photolithography using a transparent film and an office printer. The enzymatic hydrolysis of the substrate $p$-nitrophenyl phosphate (PNPP) by ALP produces p-nitrophenol, which was quantified by square wave voltammetry (SWV) and cyclic voltammetry (CV). Untreated human blood (UHB), human serum (HS), foetal bovine serum (FBS), and many other media provide a platform for ALP detection via this technique, which has a linear range of 5-250 U/L. The standard colorimetric analysis method was performed to further confirm the results.

Keywords: Alkaline phosphatase; Electrochemical sensor; Reperfusion injury; Electrode modification

\section{FULL TEXT}

(C) 2017 The Authors. Published by ESG (www.electrochemsci.org). This article is an open access article distributed under the terms and conditions of the Creative Commons Attribution license (http://creativecommons.org/licenses/by/4.0/). 